\title{
Effect of Doped and Undoped POMA on the Morphology and Miscibility of Blends with Poly(vinylidene fluoride) (PVDF)
}

\author{
I.S. Rocha ${ }^{a}$, L.F. Malmonge ${ }^{b}$, L.H.C. Mattoso ${ }^{c}$, R. Gregorio Jr. ${ }^{a^{*}}$ \\ ${ }^{\mathrm{a}}$ Universidade Federal de São Carlos, Depto. de Engenharia de Materiais, \\ C.P. 676, 13565-905 São Carlos - SP, Brazil \\ ${ }^{\mathrm{b}}$ Universidade Estadual Paulista, Depto. de Física e Química, \\ 3585-000 Ilha Solteira - SP, Brazil \\ ${ }^{\mathrm{c}}$ Empresa Brasileira de Pesquisa Agropecuária, \\ Centro Nacional de Pesquisa e Desenvolvimento de Instrumentação Agropecuária, \\ C.P. 741, 13560-970 São Carlos - SP, Brazil \\ *e-mail: gregorio@power.ufscar.br
}

Received: February 27, 1998; Revised: August 11, 1998

\begin{abstract}
The effect of the addition of small amounts of doped and undoped poly(o-methoxyaniline) (POMA) on the morphology of melt crystallized PVDF was investigated by means of polarized light optical microscopy. Undoped POMA (POMA-EB) inhibits nucleation and growth of non ringed spherulites, partially formed by the polar $\gamma$ phase, whereas POMA doped with toluene sulfonic acid (POMA-TSA) favors this process. Moreover, the doping of POMA increases the miscibility between the components of the PVDF/POMA blends, resulting in more homogeneous films. A possible cause of this miscibility increase and for the favoring of the polar $\gamma$ phase, is the higher polarity of the POMA chains as a result of the doping.
\end{abstract}

Keywords: $P O M A, P V D F$, conducting polymers

\section{Introduction}

Poly(vinylidene fluoride) (PVDF) is one of the polymers that has attracted much scientific and technological interest during the last two decades, partly due to the important pyro and piezoelectric properties it presents. These properties combined with its excellent mechanical properties and processing facility allow numerous technological applications for this polymer. Another characteristic that makes PVDF different from other polymers is its polymorphism, enabling crystallization in at least four distinct phases, known as $\alpha, \beta, \gamma$ and $\delta$. A brief description on the structure and obtaining of each one of these phases, as well as the possible conversions among them, has been given by Lovinger ${ }^{1}$, and more recently by Gregorio Jr. and coworkers $^{2}$. The morphology of both solution and melt crystallized PVDF has also been investigated ${ }^{2}$. It is common knowledge that crystallization from the melt at temperatures above $150{ }^{\circ} \mathrm{C}$ results in two distinct types of spherulites: ringed and non ringed. Increasing crystal- lization temperature decreases the nucleation rate and increases the growth rate, for both types of spherulites, leading to a reduction in the amount and an increase in the dimensions of these structures ${ }^{2}$.

Conducting polymers and their blends and composites have drawn the attention of several workers, both for their scientific importance in understanding the mechanisms responsible for the elevated electrical conduction and their potential in technological applications ${ }^{3}$. Previous investigations ${ }^{4}$ showed that blends of PVDF with poly(omethoxyaniline) (POMA) are very promising as conducting materials. In relation to other known conducting blends they present higher electrical conduction and lower percolation composition ${ }^{5}$. Nevertheless, little attention has been given to the study of the effect of POMA and its doping on the crystallization and morphology of PVDF in PVDF/POMA blends. In this work we present the effect of low concentration additions ( $2 \%$ ) of doped and undoped 
POMA on the morphology of PVDF crystallized from the melt and on the miscibility between the blend components.

\section{Materials and Methods}

POMA was chemically synthesized with ammonium peroxydisulfate in a $0.1 \mathrm{M} \mathrm{HCl}$ aqueous solution at $0{ }^{\circ} \mathrm{C}$, as described elsewhere ${ }^{6}$. Deprotonation was carried out with $0.1 \mathrm{M}$ ammonium hydroxide for $16 \mathrm{~h}$ at room temperature, resulting in dedoped POMA, known as emeraldine base (POMA-EB). Next, the resulting polymer was dried under dynamic vacuum for $24 \mathrm{~h}$ at room temperature. PVDF was purchased from Elf Atochem (Foraflon ${ }^{\circledR} 4000 \mathrm{HD}$ ) in granular form and used as received. For the blend preparation, stock solutions of POMA-EB ( $2 \%$ weight/volume) and of PVDF (18\% wt/v ) were separately prepared in dimethylacetamide (DMA). Doping of POMA was accomplished by adding toluene sulfonic acid (TSA) to the POMA-EB/DMA solution, in order to obtain a doping level of $50 \%$ (POMA-TSA) ${ }^{5}$. No insoluble fractions have been observed during solution filtering. Films $8 \mu \mathrm{m}$ thick of pure PVDF and of 98/2 wt\% PVDF/POMA blends with doped and undoped POMA were prepared by spreading the solution on a glass substrate previously heated to $100{ }^{\circ} \mathrm{C}$ and maintaining for $30 \mathrm{~min}$. The films obtained by this way were melted at $220^{\circ} \mathrm{C}$ for $15 \mathrm{~min}$ and, subsequently, cooled at a rate of $100{ }^{\circ} \mathrm{C} / \mathrm{min}$ to a given crystallization temperature at which they remained for $3 \mathrm{~h}$. The crystallization temperatures used varied from 150 to $165^{\circ} \mathrm{C}$. The melt and crystallization processes were carried out in a hot stage cell (Linkam - THS 600) with automatic time and temperature control. The heat treatment imposed on POMA-TSA during melt of the blend did not cause its dedoping, as confirmed by the presence of the $800 \mathrm{~nm}$ absorption in the UV-vis spectrum. Micrographs of the resulting morphologies were obtained by a polarized light optical microscope (Carl Zeiss) equipped with a $35 \mathrm{~mm}$ camera and an automatic exposure control.

\section{Results and Discussion}

Figure 1 shows the morphologies presented by the samples of (a) pure PVDF and of the blends containing POMA-EB (b) and POMA-TSA (c), crystallized at $155^{\circ} \mathrm{C}$. At this crystallization temperature pure PVDF presents a morphology predominantly formed by ringed spherulites, with few and small non ringed ones (some indicated by arrows in Fig. 1a). The blend containing POMA-EB presented exclusively ringed spherulites and several dark points associated with the presence of POMA clusters (Fig. 1b). The blend containing POMA-TSA presented a morphology composed by ringed and non ringed spherulites, yet with a larger amount of non ringed ones than that
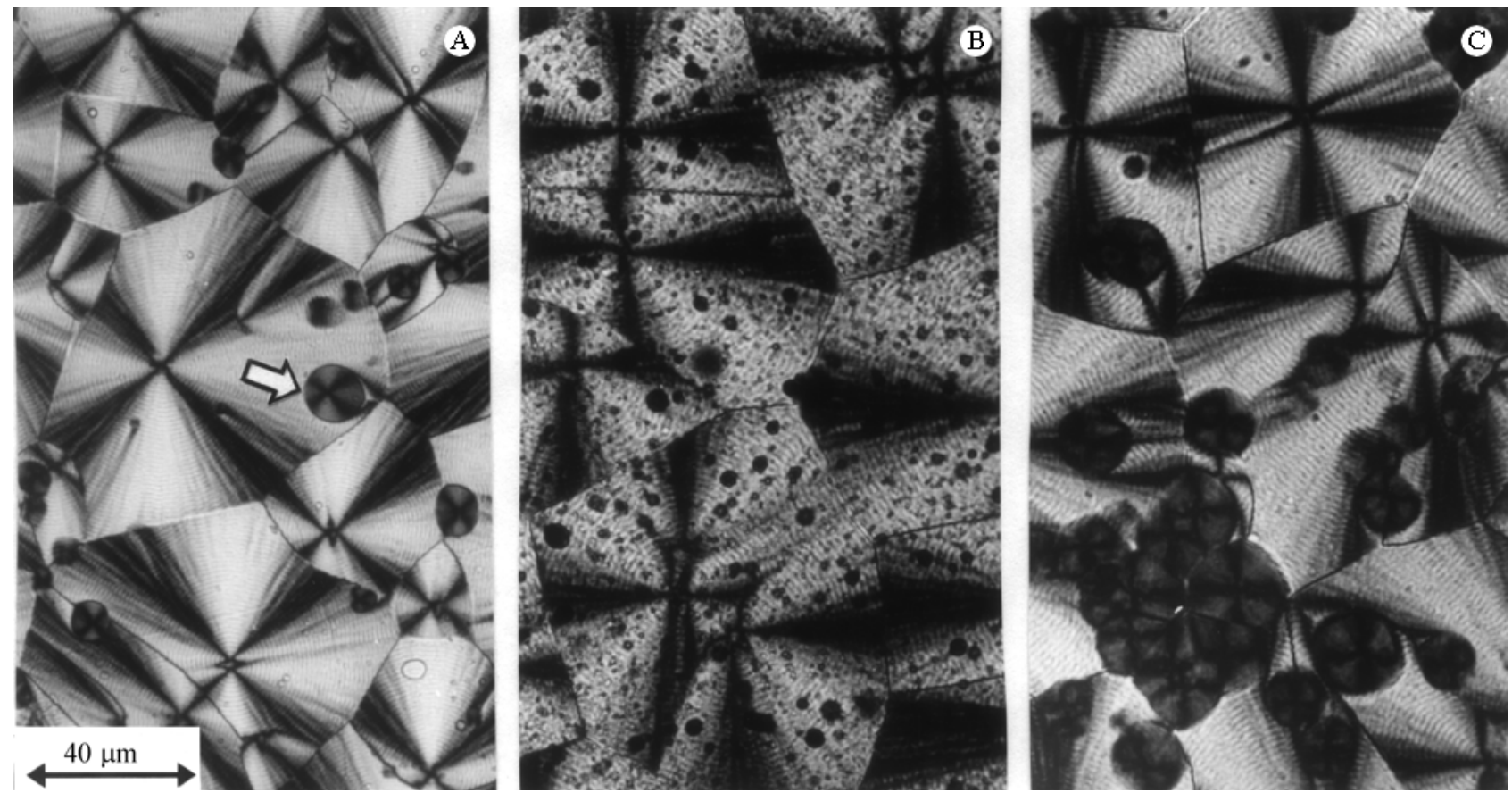

Figure 1. Optical micrographs of the morphologies presented by the samples of (a) pure PVDF and of the blends $(\mathrm{PVDF} / \mathrm{POMA}$ composition $=98 / 2)$ with POMA-EB (b) and POMA-TSA (c), crystallized at $155^{\circ} \mathrm{C}$. The arrows indicate some of the non ringed spherulites. 

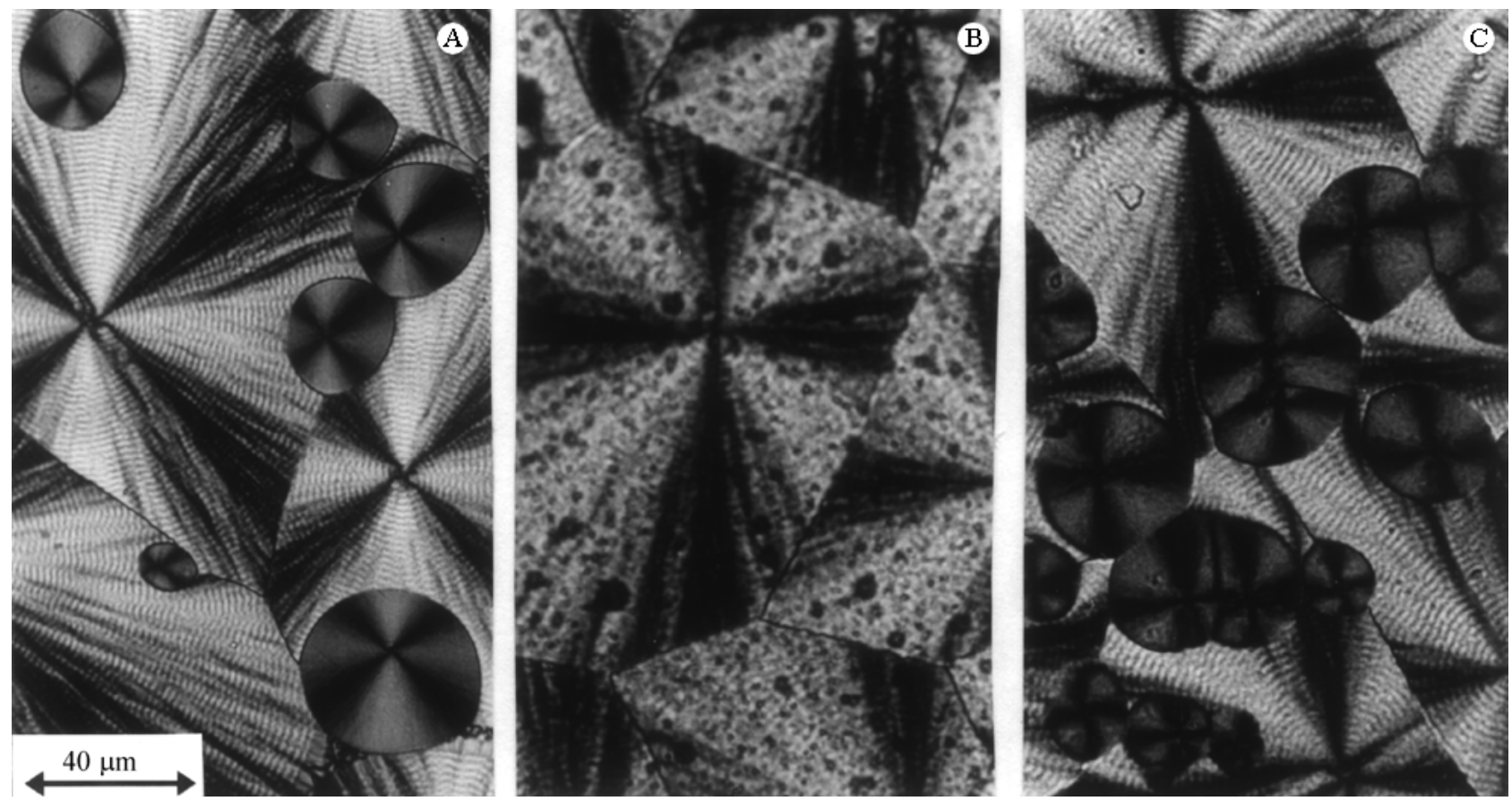

Figure 2. Optical micrographs of the morphologies presented by the samples of (a) pure PVDF and of the blends $(\mathrm{PVDF} / \mathrm{POMA}$ composition $=98 / 2)$ with POMA-EB (b) and POMA-TSA (c), crystallized at $165^{\circ} \mathrm{C}$.

presented by pure PVDF and no dark points (Fig. 1c). Similar behavior was observed in the samples crystallized at $165{ }^{\circ} \mathrm{C}$, as seen in Fig. 2. With an increase in crystallization temperature the dimensions of the two types of spherulites increases, however the result was the same as that observed at $155{ }^{\circ} \mathrm{C}$ : the blend with POMA-EB presented no ringed spherulites and many clusters (Fig. $2 b$ ); the POMA-TSA blend had a much greater number of these spherulites (Fig. 2c) in relation to pure PVDF (Fig. 2a) and no clusters. These results show that the presence of POMA-EB inhibits nucleation and growth of the non ringed spherulites, whereas POMA-TSA favors this process. Since the formation of the $\gamma$ phase takes place in these spherulites, it may be stated that POMAEB inhibits and POMA-TSA favors crystallization of this polar phase. A possible cause for such behavior may be the higher miscibility of POMA-TSA with PVDF, evidenced by the absence of POMA clusters in the blends. Previous works ${ }^{4,7}$ have shown that organic dopants may induce miscibility between blend components composed of a polyaniline derivative and a conventional polymer. In our case, considering the polarity of PVDF, this higher miscibility may have occurred due to the increase in polarity of the POMA molecular chains caused by the dopant. The more intimate mixture between PVDF and POMA and the higher polarity of POMA-TSA, may have been responsible for favoring crystallization of the polar $\gamma$ phase in detriment to the non polar $\alpha$ phase.

\section{Conclusions}

Addition of a small amount of POMA considerably affects the morphology of PVDF crystallized from the melt and the dopant exerts a strong influence on this process. Undoped POMA inhibits formation of non ringed spherulites, which contain the polar $\gamma$ phase, whereas that doped with TSA favors this formation. Moreover, doping enhances miscibility of the blend, producing more homogeneous films and indicating the important role of the dopant as compatibilizing agent.

\section{Acknowledgments}

The authors thank FAPESP and CNPq for their financial support.

\section{References}

1. Lovinger, A.J. Developments in Crystalline Polymers, Basset, D.C., ed., Applied Science Publications Ltd., London, 195, 1981.

2. Gregorio Jr., R.; Cestari, M.; Nociti, N.C.P.S.; Mendonça, J.A.; Lucas, A.A. The Polymeric Materials Encyclopedia: Synthesis, Properties and Applications, CRC Press Inc., USA, p. 7128-38, 1996. 
3. De Paoli, M.A. Organic Conductive Molecules and Polymers, v. 2, Nalwa, H.S., ed., John Wiley, New York, 1997.

4. Mattoso, L.H.C.; Malmonge, L.F. Proc. Internat. Conf. Sci. Technol. Synthet. Met., Seul-Coréia, julho/1994, p. 380.
5. Malmonge, L.F. PhD Thesis, IFSC- Universidade de São Paulo, 1996.

6. Mattoso, L.H.C., Faria, R.M., Bulhões L.O.S.; MacDiarmid, A.G., J. Polym. Sci: Polym. Chem., v. 32, p. 2147, 1994.

7. Cao, Y.; Smith, P.; Heeger, A.J. Synth. Met., v. 55-57, p. $3514,1993$. 